\title{
DAS SOGENANNTE FREUDENHAUS ZU EPHESOS Neues Zur Hausentwicklung des Komplexes
}

\section{Topographie}

Der Hauskomplex, welcher Teil einer geschlossenen Verbauung ist (Insula M/1) ${ }^{1}$, die von der Kuretenstraße bis zum Theater reicht, liegt am südwestlichen Hangfuß des Panayırdağ. Die östliche Begrenzung stellt die Akademiegasse dar, die auf deutlich höherem Niveau liegt. Um ein nur leicht von Westen nach Osten ansteigendes Terrain zu erhalten, das besser bebaut werden konnte, wurde der Hang in großem Umfang abgegraben; dies zeigen mächtige Hangstützmauern, vor allem im Mittelteil der Insula.

Die Lage der Insula kann als äußerst prominent bezeichnet werden: an ihrem südlichen Ende paßt sie sich dem Verlauf des alten Prozessionsweges an und öffnet einen Zwickel nach Südosten, die Begrenzung im Norden erfolgt durch eine Stiegengasse, die von der Marmorstraße bis hin zur Akademiegasse verläuft².

\section{Forschungsgeschichte}

Franz Miltner grub in den fünfziger Jahren des 20. Jahrhunderts im Zuge sehr intensiver Kampagnen die sog. Scholastikiatherme aus sowie im Jahr 1957: »An der Scholastikiatherme ... die ebenerdigen Räume zwischen der Marmorstraße und dem schon 1956 freigelegten Substruktionsgang $\aleph^{3}$. Miltner deutete den vielräumigen, in sich geschlossenen Komplex entsprechend den Angaben der Bauinschrift der Latrine als öffentliches Freudenhaus und versuchte daher, den Befund in diesem Sinne zu interpretieren ${ }^{4}$. Werner Jobst beschäftigte sich in den siebziger Jahren nochmals mit dem Komplex; er legte ein Mosaik und den Teil eines Stylobaten frei und erstellte eine erste Baubeschreibung, die allerdings auf den östlichen Bereich der Anlage beschränkt blieb. Jobst setzte sich auch mit der von Miltner angeführten Bauinschrift auseinander und kam zu folgendem Schluß: »Die an dieser höchst zentral gelegenen Stelle von Ephesos durchgeführte Nachuntersuchung hat also erwiesen, daß unweit des für seine vielen offiziellen Bauwerke berühmten sog. Embolos ein ’öffentliches Freudenhaus nicht gestanden sein kann. $\ll^{5}$

Im Zuge der in den Jahren 2000 und 2001 unternommenen Bauuntersuchung (Abb. 1. 2) erwiesen sich die größten Teile der Insula M/1 - sieht man von der Latrine ab - als von der 'Scholastikiatherme' autonom. Doch die anfängliche Bebauung ist ohnehin früher anzusetzen als

\footnotetext{
1 Die Grundlage des hier Vorgelegten bildet die ungedruckte Diplomarbeit des Verf.: D. Boulasikis, Das Freudenhaus zu Ephesos. Untersuchung zur Baugeschichte eines Hauskomplexes der Insula M/1 an der Marmorstraße (ungedr. Mag. Wien 2001).

2 F. Miltner, XXII. Vorläufiger Bericht über die Ausgrabungen in Ephesos, ÖJh 44, 1959, Beibl. 243-314 Abb. 120. - Bezeichnung Akademiegasse nach D. Knibbe - H. Thür (Hrsg.), Via Sacra Ephesiaca II, BerMatÖAI 6 (1995) 84 Abb. 48.

3 F. Miltner, XXIII. Vorläufiger Bericht über die Ausgrabungen in Ephesos, ÖJh 44, 1959, Beibl. 315.

4 Ebenda 318 f.; zur Bauinschrift der Latrine: F. Miltner, XXI. Vorläufiger Bericht über die Ausgrabungen in Ephesos, ÖJh 43, 1956-58, Beibl. 21 f.

5 W. Jobst, Das 'öffentliche Freudenhaus' in Ephesos, ÖJh 51, 1976/77, 84.
} 


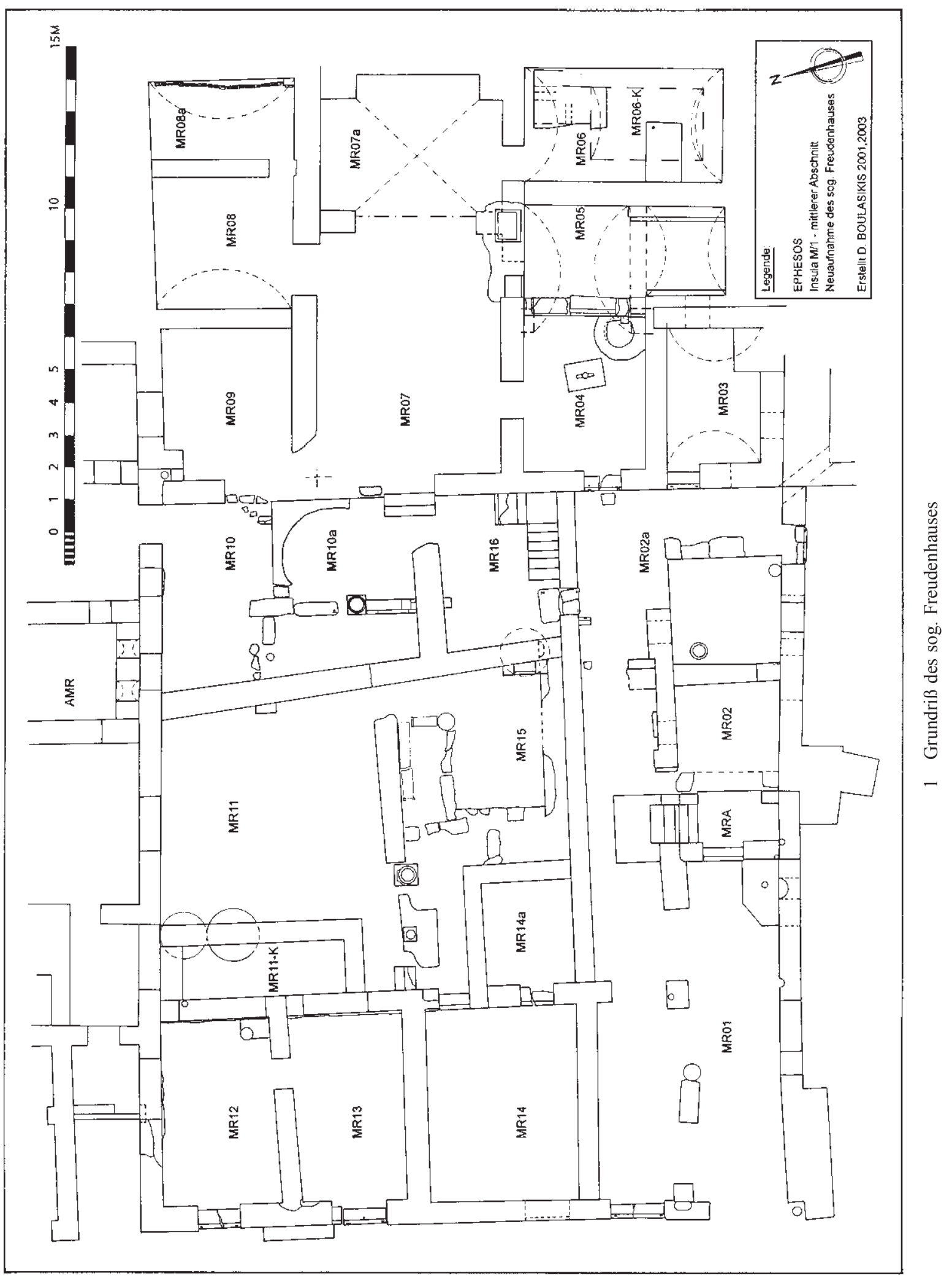


die oberhalb der Insula gelegene Thermenanlage in ihrer heute sichtbaren Form ${ }^{6}$. Die Theorie schließlich, ein in der Insula gelegenes Freudenhaus anzunehmen, konnte bereits von Jobst widerlegt werden. Zu klären blieb, welche Grundrisse die einzelnen Phasen der Bebauung innerhalb des vorgegebenen Rahmens der Insula M/1 ausprägten und schließlich, wie sich die einzelnen Bauphasen zueinander verhielten ${ }^{7}$.

\section{Baugeschichte}

\section{Phase: Peristylhaus}

Die Süd-, Ost- und Nordbegrenzungen des rekonstruierten Peristylhauses erster Phase (Abb. 4) im Mittelteil der Insula M/1 sind durch Einschnitte in den Hangfuß des Panayırdağ zu erkennen. Die Nordseite des Hauses wird durch eine Stichgasse, die im rechten Winkel von der Marmorstraße gegen den Hang läuft, von der nördlich anschließenden Verbauung der Insula abgegrenzt; an der Westseite liegen Tabernen, die sich zur Marmorstraße hin öffnen (Abb. 3).

Die Verbauung der ersten zu erfassenden Phase wird von einem nahezu quadratischen Peristyl dominiert. Der Peristylhof verfügt an allen vier Seiten über Umgänge, an die wiederum

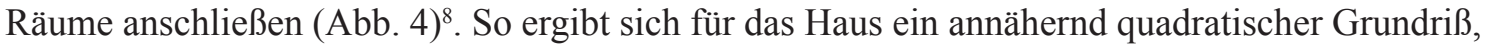

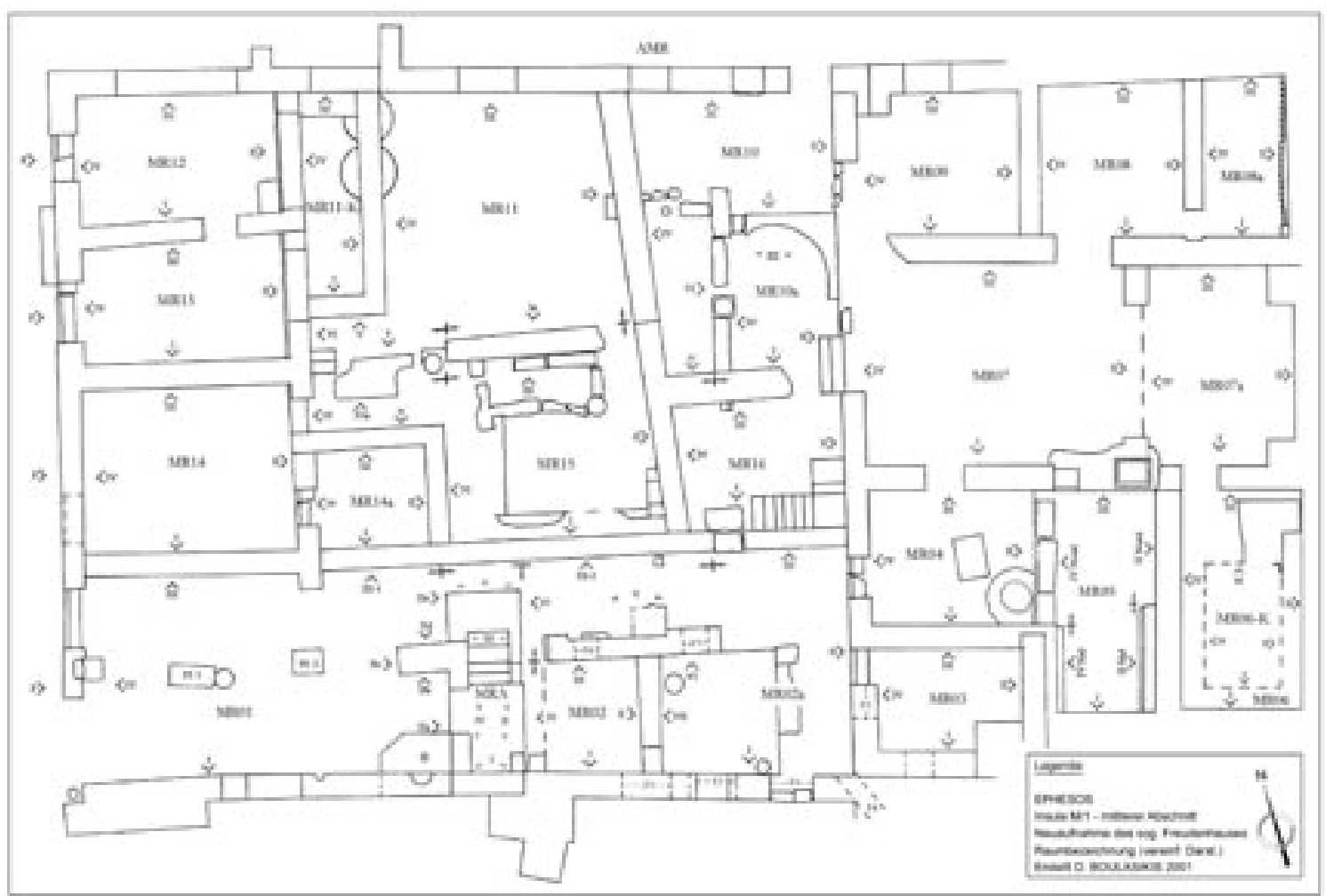

2 Bezeichnung der Räume und Raumgrenzen, vereinfachte Darstellung

${ }^{6}$ W. Jobst datiert die Funde aus den von ihm angelegten Sondagen vom Hellenismus bis in die frühe Kaiserzeit: Jobst (Anm. 5) 73.

7 Für eine umfangreichere Darstellung s. o. Anm. 1.

8 s. Abb. 4, die blauen Bereiche kennzeichnen den gesicherten Bestand aus der Phase 1. Abb. 1 und Abb. 2 geben die bis jetzt eingehend untersuchten Räume wieder, die vorhandenen Mauerzüge der nördlichen Seite des Peristylhauses sind in Abb. 1 nur teilweise angegeben, werden in den Rekonstruktionen (Abb. 4-6) aber berücksichtigt. Für die Mauerzüge der nördlichen Seite des Peristylhauses bis zur Stichgasse s. o. Anm. 2. 


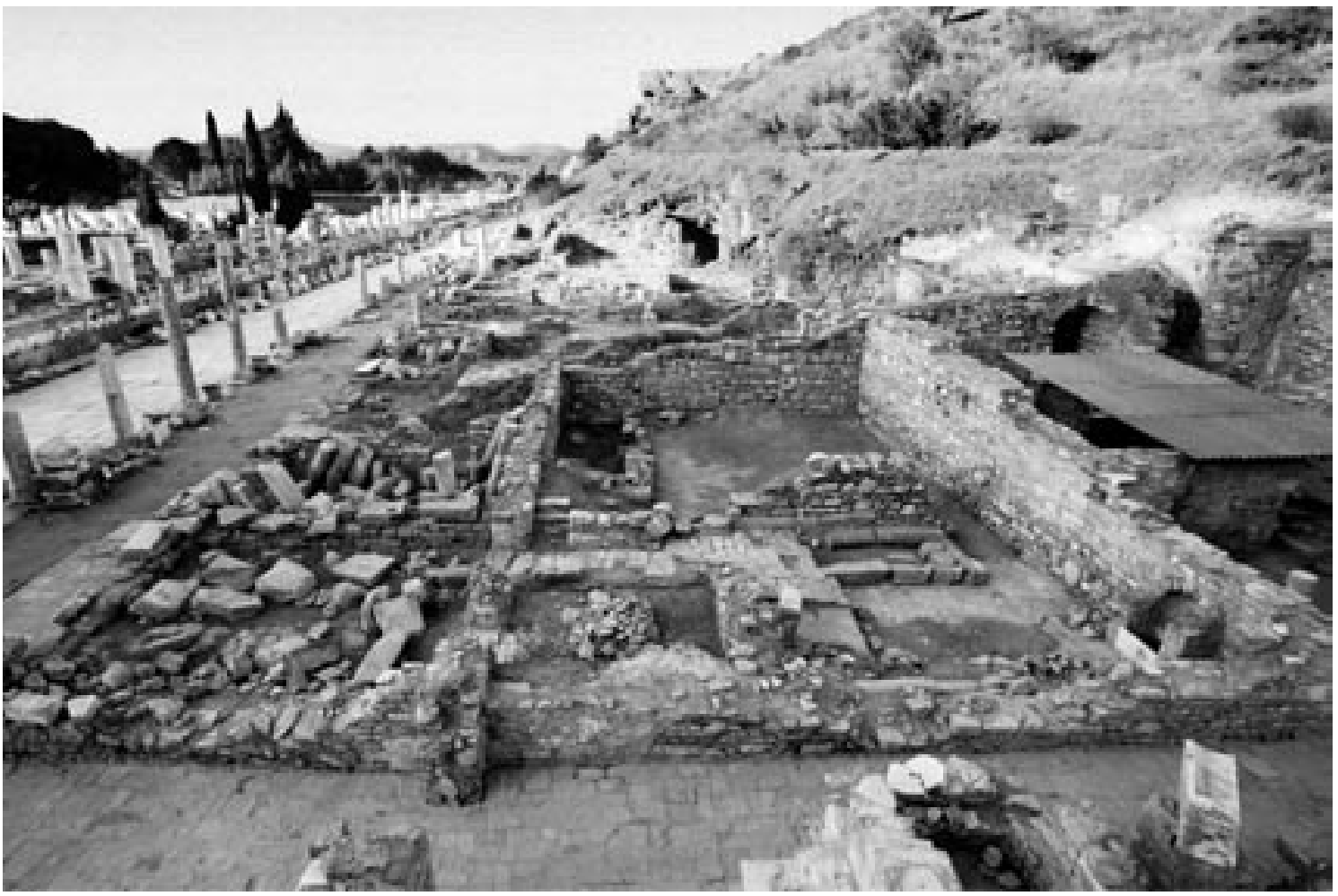

3 MR01, MR11-MR15: Überblick nach Norden, Erhaltungszustand 2000

seine Seitenlängen betragen $26.1 \times 28.45 \mathrm{~m}$, daraus resultiert eine Grundfläche von etwa $742.5 \mathrm{~m}^{2}$. Der zentrale, von Säulen umstandene Hof weist eine Fläche von $63.56 \mathrm{~m}^{2}$ auf, seine Seitenlängen betragen $7.77 \times 8.18 \mathrm{~m}$. Die umlaufenden Säulengänge messen zwischen 3.1 und $3.2 \mathrm{~m}$ Breite, der Südgang ist mit etwa $2.7 \mathrm{~m}$ etwas schmäler zu rekonstruieren'. Die Maße des Stylobaten sind über Fragmente, größtenteils in situ, gut zu erschließen. Er bestand aus $66 \mathrm{~cm}$ breiten, grauen, grünblau marmorierten Blöcken mit polierter Oberfläche. Die Jochweite der Säulenstellung auf dem Oststylobat kann über eine ionische Säulenbasis und eine Säulenstandspur in der Nordostecke des Stylobaten mit 2.94,5 m bestimmt werden, woraus sich für die Ostseite 4 Säulen ergeben und so ein Peristyl mit 12 Säulen zu rekonstruieren ist.

Der Haupteingang des Hauses lag wohl an der Marmorstraße, von hier gelangte man über ein Vestibül in die Südwestecke des Peristylumganges. Während dieser Zugang bis in die späteste Phase einigermaßen unverändert erhalten geblieben ist, veränderte sich die Situation an der Nordseite des Hauses grundlegend. Durch die sekundäre Überbauung der Stichgasse und der damit verbundenen Verwischung der Hausgrenze kann hier ein Eingang nicht mehr zweifelsfrei festgestellt werden, man darf aber wohl davon ausgehen, daß ein solcher bestanden hat ${ }^{10}$.

An jeden Peristylgang grenzen drei Räume an; die in den Ecken gelegenen wurden vermutlich über den jeweiligen Nebenraum erschlossen ${ }^{11}$. Der Zugang zum zentralen Raum an der

9 Der Absprache am Münchner Lehrstuhl für Baugeschichte und Bauforschung folgend, steht bei allen Maßangaben der Punkt immer nach Metern (z. B. 4.12 m), das Komma nach Zentimetern (z. B. 4.12,5 m).

10 Vgl. D. Pinkwart - W. Stammnitz, Peristylhäuser westlich der unteren Agora, AvP XIV (1996) bes. 41. Auch in Ephesos ist eine solche Lösung belegt; C. Lang-Auinger schreibt über das von ihr publizierte Peristylhaus: »Der, wie im Hellenismus üblich, an der ruhigen Seitenstraße liegende Haupteingang führt direkt in den nördlichen Peristylgang ...«: C. Lang-Auinger, Hanghaus 1 in Ephesos. Der Baubefund, FiE VIII 3 (1996) 183.

11 Dafür scheint die in einer späteren Phase unverändert übernommene Türöffnung zu MR03 zu sprechen (s. Abb. 1. 2, Öffnung in MR03 IV). 


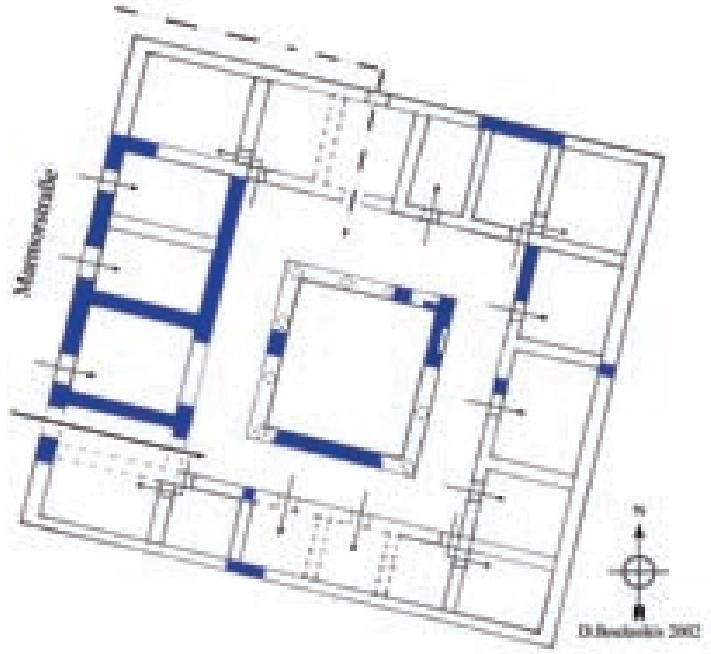

4 Phase 1

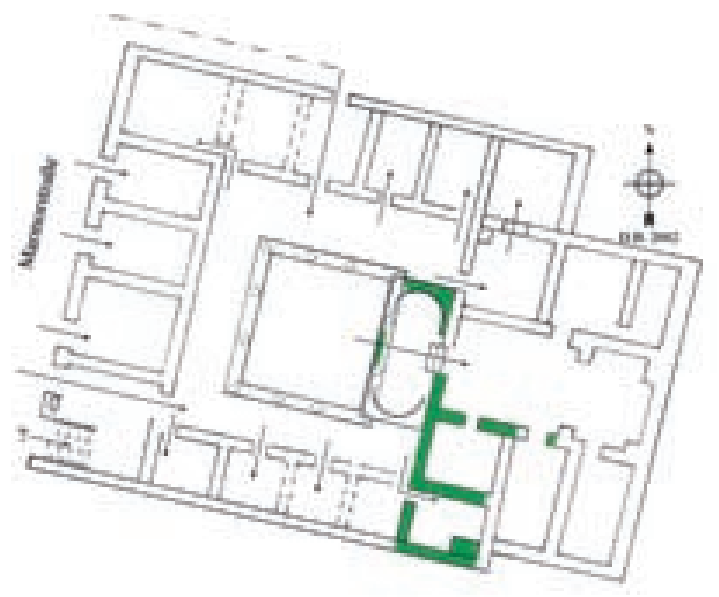

6 Phase 3

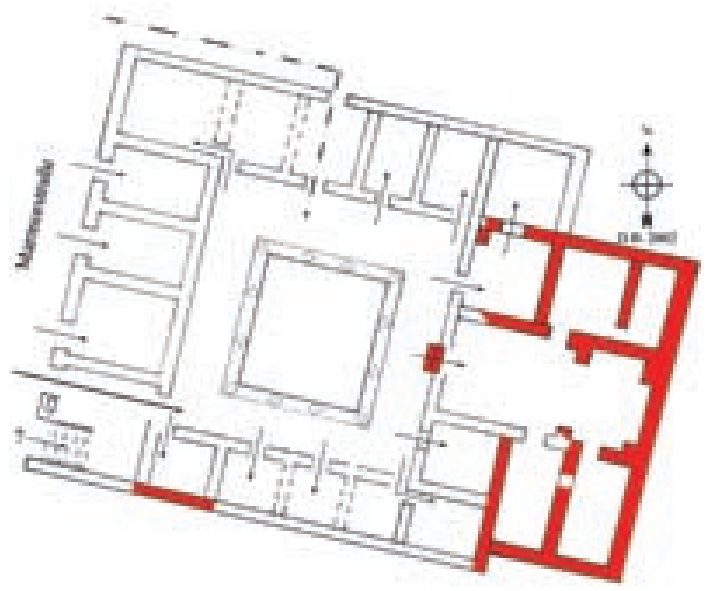

5 Phase 2

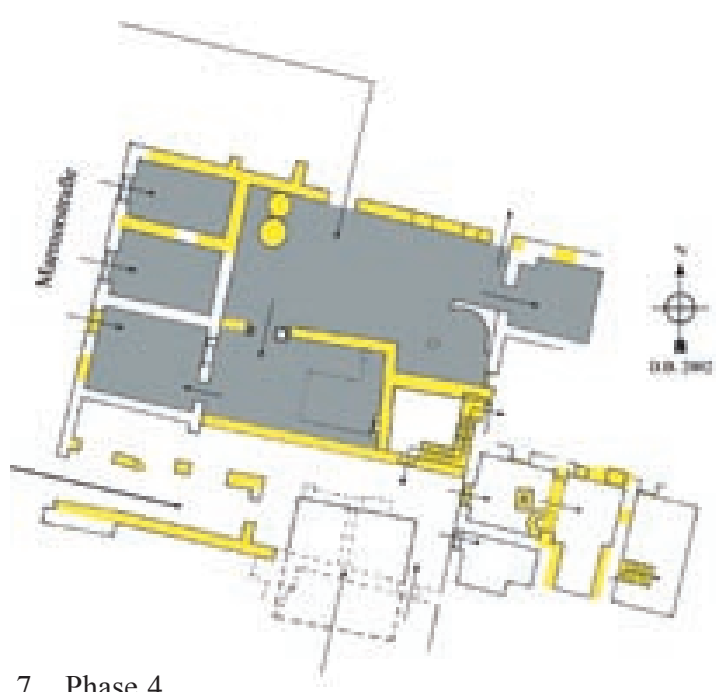

7 Phase 4

Ostseite des Hauses ist gut nachweisbar, auch die Türöffnung zum Raum am Nordende des Ostganges ist gut zu erschließen (Abb. 1. 2) ${ }^{12}$. Während die Raumverteilung des östlichen Hausbereiches über gesicherte Zugänge rekonstruiert werden kann, ist die Gliederung der südlichen Räume des Hauses anhand des Restes einer Trennmauer zu gewinnen ${ }^{13}$. Erhalten und für die Rekonstruktion der ersten Bauphase relevant sind des weiteren die westliche Hofmauer, die als Fundament für spätere Aufmauerungen genützt wurde, sowie im Süden und Norden Teile der Hausmauer ${ }^{14}$.

Grundlage der Beobachtungen ist, neben dem Stylobaten, der in sich geschlossene Mauerbefund. Das verbaute Material besteht aus lokal anstehendem, grauem Marmor vom Panayırdağ, wie er bei der Anlage der in den Hang geschnittenen Insula reichlich anfiel. Die Mauern wurden aus zugerichteten Bruchsteinen und ohne Ziegel hergestellt, der Fugenmörtel enthält ausreichend Kalk und ist dementsprechend fest.

\footnotetext{
12 s. Abb. 1. 2, Öffnung in MR10a II sowie Öffnung in MR10 II.

13 s. Abb. 1. 2, MRA I, MRA IV.

14 s. Abb. 1. 2, MR12 II, MR13 II.
} 
An zwei Mauerresten in etwa gleichem Abstand westlich und östlich des Stylobaten, welche die oben beschriebene Struktur aufweisen, hat sich direkt auf das Mauerwerk aufgetragener Feinputz mit einer darüberliegenden kräftigroten Farbschicht erhalten. Die Farbspuren können aufgrund ihrer Gleichartigkeit als Reste einer Bemalung, mit der die Hofwände ausgestattet waren, gedeutet werden ${ }^{15}$.

Mit der ersten Phase der Bebauung kann mit Sicherheit auch der Plattenbelag des Peristylhofes in Verbindung gebracht werden, der in einem Mörtelbett verlegt war; zwei Bruchstücke der großformatigen Platten aus grauem Marmor haben sich erhalten ${ }^{16}$.

\section{Phase: 'Peristyl-Atrium-Haus'}

In der nachfolgenden Phase der Bebauung wird eine Hauserweiterung nach Osten gegen den Hang hin vorgenommen. Die Erweiterung wird von einer neugeschaffenen, axialen Raumfolge östlich des Peristyls beherrscht, an die südlich und nördlich Räume anschließen (Abb. 5) ${ }^{17}$. Der Hauptzugang zu den neu angebauten Räumlichkeiten erfolgt über den bereits bestehenden Eingang zum mittleren Raum am Ostgang des Peristyls; dessen Rückwand, zugleich die ehemalige Hausgrenze, wird im Zuge der Erweiterung abgebrochen ${ }^{18}$ : auf diese Weise entsteht ein länglicher, etwas über $41 \mathrm{~m}^{2}$ großer Raum MR0 $7^{19}$. Östlich axial anschließend wird ein mit einem Kreuzgewölbe versehener Raum geschaffen, der nach Westen hin geöffnet bleibt (MR07a) ${ }^{20}$. Südlich liegen die mit Tonnen überwölbten Räume MR06 und MR05, die über Eingänge mit MR07 bzw. MR07a verbunden sind. Der Raum MR06, in der Südostecke des Hauses gelegen, war zusätzlich unterkellert, eine Stufenfolge führte in einen $9.3 \mathrm{~m}^{2}$ großen Raum, der $2.15 \mathrm{~m}$ unter dem Bodenniveau von MR06 liegt. Nördlich anschließend an die zentralen Räume MR07 und MR07a liegen MR08 und MR08a. Während MR08 direkt von MR07 erschlossen wird, konnte MR08a, in der nordöstlichen Ecke des Hauses, nur über MR08 betreten werden.

Da der Mauerbefund in den Räumen östlich des Peristyls weitestgehend in sich geschlossen ist, scheint die Annahme einer gemeinsamen Entstehung in einer weiteren, von der ersten Phase deutlich abgrenzbaren Baustufe gesichert zu sein. Das Mauerwerk der Phase 2 wurde als sorgfältiges opus mixtum ausgeführt: Bruchsteinreihen, vorwiegend aus lokalem Marmor, aber auch zugerichtete Spolien, wechseln mit ein- bis vierlagigen Ziegelausgleichsschichten ab. Der verwendete Mörtel ist zumeist hart, er enthält Serecitsplitt als Zuschlagstoff. Die zentral gelegenen Räume dieser Phase waren mit Marmorwandverkleidungen ausgestattet, peripher angelegte Räume mit weißem Verputz versehen.

Für eine Hauserweiterung nach Osten sprechen neben dem Mauerbefund auch die Stärke und Lage einer von Werner Jobst aufgenommenen Mauerstruktur. In den Plan seiner Nachgrabung und in seiner Ansicht der Nordwand ${ }^{21}$ sind im Boden liegende Mauern eingezeichnet, die besondere Beachtung verdienen. Sie laufen in Nord-Süd-Richtung und stehen parallel zueinander; die westliche ist etwa $70 \mathrm{~cm}$ breit und kann als Sockel der nur mehr teilweise erhaltenen Mauer des Ostganges des Peristyls ${ }^{22}$ interpretiert werden. Die östliche der beiden von Jobst aufgenommenen Mauern liegt in $4.30 \mathrm{~m}$ Abstand von der westlichen und weist eine Breite von etwa $90 \mathrm{~cm}$ auf. Die beachtliche Mauerbreite gab Anlaß, nach Parallelen in der näheren Umgebung

\footnotetext{
15 s. Abb. 1. 2, Baufuge zwischen MR01 III-1/III sowie MR10 II.

16 s. Abb. 1. 2, Boden vor MR11 Ia.

17 s. Abb. 5, die roten Bereiche kennzeichnen erhaltene, in der 2. Phase errichtete Gebäudeteile.

18 s. Abb. 5; s. u.

19 s. Abb. 1. 2, MR07.

20 s. Abb. 1. 2, MR07a.

${ }^{21}$ s. Jobst (Anm. 5) 68. 74 (Abb. 9. 17).

22 s. o.; s. Abb. 4, Phase 1.
} 


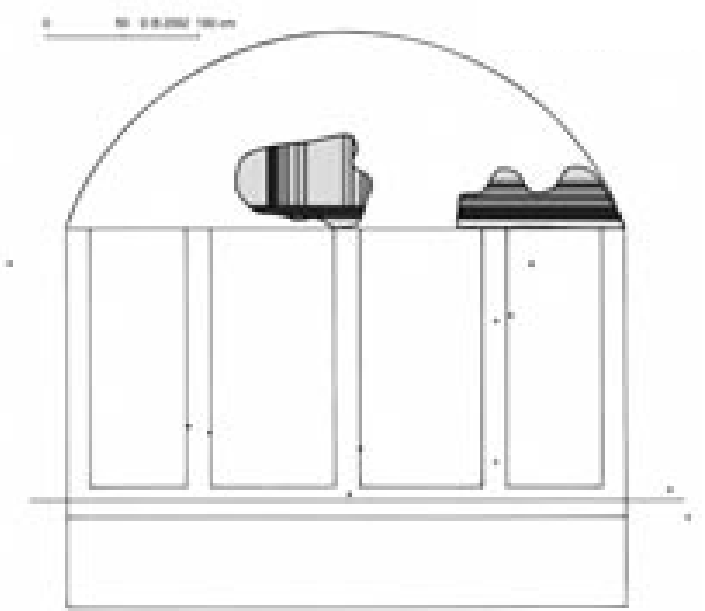

8 MR07a II. Marmorplattenverkleidung mit bemalter Lünette

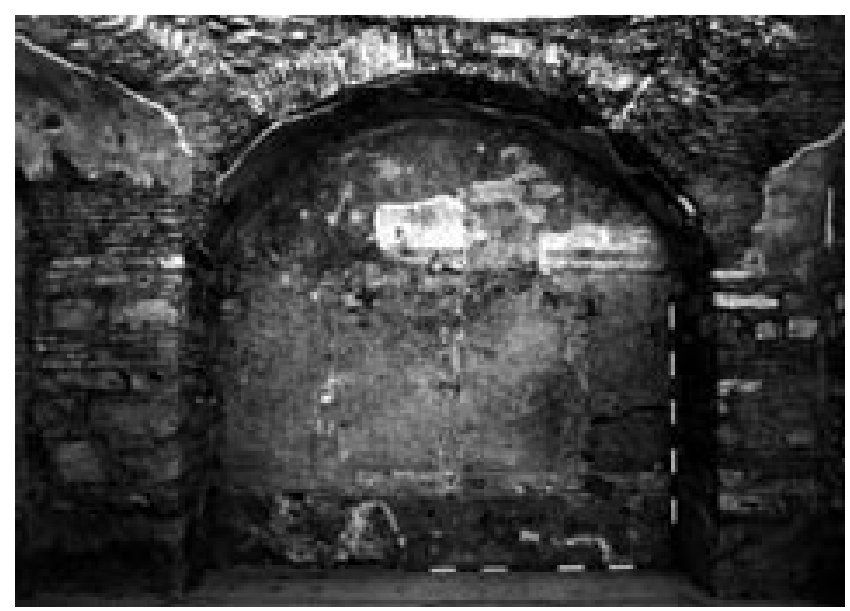

9 MR07a II. Erhaltungszustand 2000

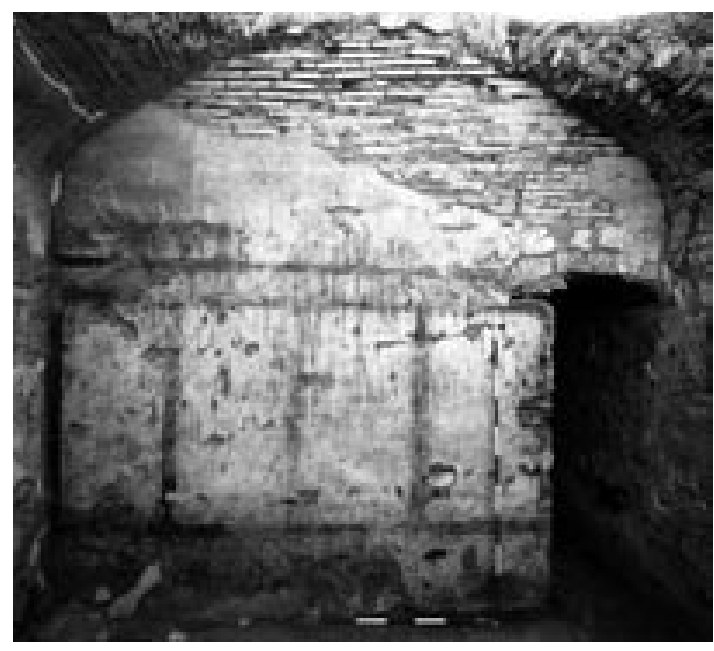

10 MR08 II. Erhaltungszustand 2000

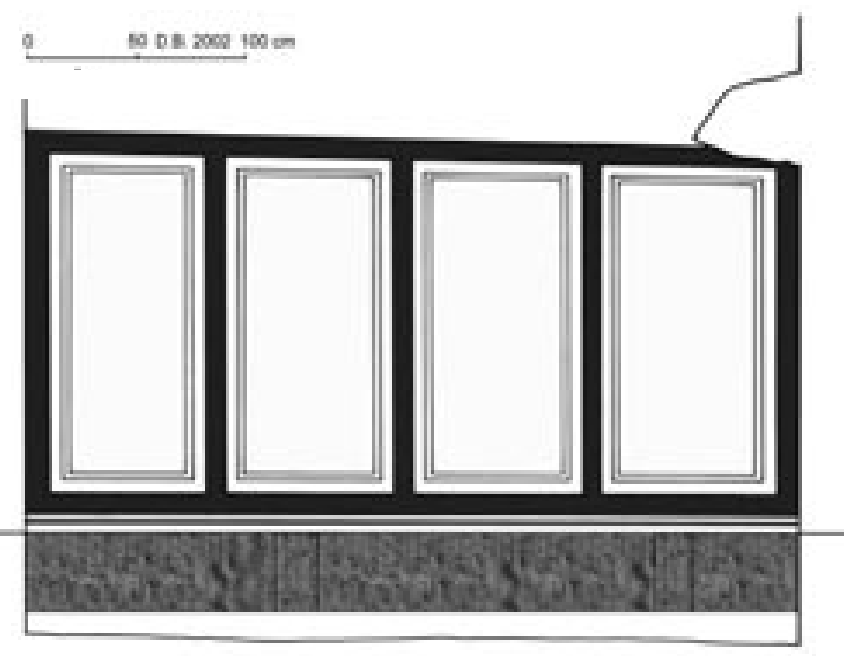

11 MR08 II. Rekonstruktion der Malerei, 3. Phase

des Hauses zu suchen. Sie fanden sich, und zwar in den Mauern des nördlich des Hauses liegenden, schlauchartigen Raumes, dem Ende der oben beschriebenen Stichgasse sowie in einer Mauer, die von Norden her, parallel zur Marmorstraße, auf diesen Raum zuläuft. Bei dieser Mauer handelt es sich eindeutig um eine Terrassenmauer, die gegen den Westhang des Panayırdağ errichtet wurde. In diesem Sinn ist m. E. auch die heute nicht mehr sichtbare, MR07 in NordSüd-Richtung durchlaufende Mauer zu deuten und als Teil der Hausmauer, die das Peristylhaus der ersten Phase gegen den Hang abgrenzte, zu verstehen.

Die zweite Bauphase manifestiert sich im Raum MR07 in Teilen der nördlichen Raumbegrenzung sowie in den Mauerpfeilern des östlich anschließenden Raumes MR07a. MR07 war mit Marmorplatten ausgelegt, Reste haben sich in der Südwest- und Nordostecke erhalten. Die den Raum nach Süden und Westen begrenzenden Mauern sind aufgrund ihrer Struktur und Baufugen einer nachfolgenden Phase zuzuordnen. Gut belegt werden kann die zweite Phase im östlichen Raum MR07a, der von einem massigen Kreuzgewölbe überspannt war, das auf Mauerpfeilern ruhte. Nach Westen blieb der Raum offen, in der Südwand liegt ein Eingang nach MR06. An Wänden wie Pfeilern war eine Marmorplattenverkleidung angebracht, die einen Aufbau in drei Zonen aufwies, oberhalb in den Lünetten wurde farbige Streifenmalerei von Stuck- 
leisten gerahmt (Abb. 8. 9). Am Boden war ein polychromes Mosaik verlegt, das bereits von Franz Miltner und später von Werner Jobst publiziert wurde ${ }^{23}$.

Miltner bezeichnete den Raum MR07 als Atrium und äußerte die Vermutung, es habe »ein seichtes Impluvium $\ll^{24}$ existiert, Jobst will sich »mangels erhaltener Bauelemente«, wie er schreibt, nicht festlegen. Er zweifelt, »daß die Absicht bestanden hat, ein solches [Atrium] mitten unter geschlossenen Wohnräumen zu errichten $\ll^{25}$. Für einen Raum, der zumindest teilweise nach oben geöffnet war - will man ihn nun als Atrium bezeichnen oder nicht -, spricht aber die Anlage von MR07a; er blieb an seiner Westseite offen und war unbestreitbar als Blickfang angelegt, dessen aufwendige Ausstattung seine repräsentative Bedeutung unterstrich. Dieses sog. Tablinum war auf eine Lichtquelle angewiesen, die seine Ausstattung zur Geltung bringen konnte; was liegt also näher, als den Raum MR07 mit einem solchen Oberlicht zu rekonstruieren?

Hier sei nun eine weitere Überlegung angeführt: Es ist evident, daß der Raum MR07, als Atrium angesprochen, nicht den Kanon erfüllt, weder liegt er in der Nähe der Straße, noch entspricht das Verhältnis seiner Seitenlängen jenem, das Vitruv vorschlägt ${ }^{26}$. Ich denke aber, daß in diesem Zusammenhang der geographischen Lage von Ephesos Rechnung getragen werden muß und verstehe daher die Anlage eines atriumähnlichen Raumes als eine architektonische Rezeption italischer Vorbilder. Dabei zählt in erster Linie die Idee der Reminiszenz, die Einhaltung der Normen hingegen, die für die Vorbilder verbindlich waren, ist hier von nachgeordneter Bedeutung.

In den südlich gelegenen Räumen MR05 und MR06 haben sich die Außenmauern sowie die Trennmauer zwischen den beiden Räumen erhalten, die Türöffnung von MR07 nach MR05 wurde in der nachfolgenden Phase nahezu unverändert übernommen, der südwestliche Mauerpfeiler von MR07a erst in der letzten Nutzungsphase durch den Einbau von Nischen gestört ${ }^{27}$. Der Raum MR06 ist in beinahe ursprünglichem Zustand erhalten, seine Ausstattung war sehr einfach, man begnügte sich mit einfachem Wandverputz. Beide Räume waren mit in Ost-WestRichtung gespannten Tonnengewölben versehen. Der Kellerraum unterhalb von MR06 ist zur Gänze aus Ziegeln errichtet, er war ebenfalls verputzt. An seiner Südseite sind symmetrisch drei gleich große Nischen eingelassen.

Die nördlich von MR07 und MR07a gelegenen Räume MR08 und MR08a waren deutlich repräsentativer ausgestaltet. An den Wänden beider Räume kann eine Marmorwandverkleidung nachgewiesen werden, die analog zu MR07a in drei Zonen gegliedert war. MR08a konnte nur über MR08 betreten werden, die Trennmauer wurde in einer Zwischenphase eingezogen. Ein Tonnengewölbe, das in Nord-Süd-Richtung gespannt war, schloß die Räume nach oben hin ab. Die hangseitige Ostmauer von MR08a war mit einer Tubulatur versehen, die allerdings dazu diente, Kondensat von der Wandverkleidung fernzuhalten; ein Rohr diente der Entlüftung ${ }^{28}$. Vitruv erwähnt eine ähnliche Konstruktion ${ }^{29}$. Das obere Ende der Tubulatur muß so verschlossen gewesen sein, daß die einzelnen Stränge untereinander verbunden waren, eine Zuleitung führte entlang der Oberkante der Tubulatur zum Rohr in der südöstlichen Raumecke. Während in MR08a kein Boden nachzuweisen ist, kann für MR08 aufgrund der Estrichspuren ein Plattenboden angenommen werden.

Abschließend ergibt sich für das Haus der zweiten Phase folgendes Bild: Das Peristylhaus der ersten Phase wurde nahezu unverändert belassen, Änderungen könnte es lediglich im Eingangsbereich an der Marmorstraße gegeben haben. Die Ostseite hingegen wurde aufgebrochen,

\footnotetext{
23 Miltner (Anm. 3) 319; Jobst (Anm. 5) 69 ff.

24 Miltner (Anm. 3) 319.

25 Jobst (Anm. 5) 72.

26 Vitr. 6, 3, 3 (274 ed. Fensterbusch). Atrien sollten demnach entweder ein Breiten-Längen-Verhältnis von 3:5 oder von 2:3 oder von a:a $\sqrt{2}$ aufweisen, die Seiten von MR07 ergeben allerdings ein Verhältnis von 1,9:2,45.

28 Miltner (Anm. 3) 320. Er spricht sich für eine Heizung aus, die ein Warmbad beheizte, das Rohr deutet er als Zuleitung für Wasser.

29 Vitr. 6, 4, 1 (288 ed. Fensterbusch), Z. 10: ... et in summo habuerit exitus...
} 
das Haus um einen inneren, abseits der Straße gelegenen, repräsentativen Bereich erweitert, dessen bestimmendes Element eine zentrale Raumgruppe darstellte. Auf diese Weise wurde eine eindrucksvolle Blickachse vom Peristyl in das Innerste des Hauses geschaffen, der sich der Besucher nur schwer entziehen konnte. Auf die östlichen Bereiche des Hauses war nachweislich ein Obergeschoß gesetzt, das in etwa auf dem Niveau der Akademiestraße lag, die den Panayırdağ hinaufführt.

\section{Phase: 'Peristyl-Atrium-Haus' II}

Die dritte Phase bringt nur unwesentliche Veränderungen des Grundrisses mit sich, doch kann sie anhand von Mauerstrukturen deutlich von der zweiten differenziert werden (Abb. 6) ${ }^{30}$. Die baulichen Maßnahmen betrafen den mittleren Hausbereich, in den östlichen Peristylumgang wurde ein apsidialer Raum eingebaut, die Mauern von MR03 und MR04 entstanden neu, wie auch die Nordmauer von MR05. In die Südostecke von MR03 wurde ein mächtiger Mauerpfeiler als Substruktion einer im Oberstock angelegten Latrinenanlage eingebaut.

Bedeutsame Veränderungen gibt es in Phase 3 bei den Wandausstattungen: Wandverkleidungen werden von Malereien, die solche imitieren, abgelöst.

Die Mauern, die der dritten Phase zugeordnet werden können, wurden in opus mixtum-Technik errichtet, allerdings kommen zwischen den Bruchsteinreihen, für die nun öfter zerkleinerte Spolien (vorwiegend weißer Marmor) verwendet wurden, nur mehr ein- bis zweilagige Ziegelstreifen vor. Besonders charakteristisch ist die Verbauung von Wandverkleidungsresten, die zusammen mit Ziegeln zur Herstellung der Ausgleichsschichten verwendet wurden. Dem Fugenmörtel wurde Serecitsplitt zugeschlagen, er ist äußerst hart.

Der Raum in der Südostecke des Peristylhauses (MR03) wurde völlig neu errichtet, der Eingang orientiert sich wohl am vorhergehenden Mauerwerk. Die Wände des Raumes blieben unverputzt, ein Tonnengewölbe trägt das Obergeschoß. Der nördlich anschließende Raum MR04 konnte in dieser Phase nicht mehr direkt vom Peristylumgang aus betreten werden, seine neuerrichtete Westmauer weist keinen Eingang auf; auf diese Weise gewinnt die mittlere Türöffnung am Ostgang des Peristyls an Bedeutung, denn nun wird einzig durch diesen Eingang der gesamte an der Akademiegasse gelegene Hausbereich erschlossen. In der neuerrichteten Mauer zwischen MR07 und MR04 ist ein Durchgang vorgesehen, MR04 ist nun nur mehr von Norden her betretbar. MR05 bleibt weiterhin von MR07 aus zugänglich, der Eingang liegt jenem zu MR08 gegenüber.

Bedeutendstes bauliches Element dieser Phase ist, wie oben erwähnt, ein apsidialer Raum, der in den Ostgang des Peristyls eingebaut wird. Dieser Saal läßt sich über die noch erhaltene, aus Ziegeln errichtete, nördliche Apsis rekonstruieren, deren flache Rückseite mit dem Nordstylobat abschließt. In der Sockelzone hat sich eine Plattenverkleidung erhalten. Der Boden des Raumes ist mit einem polychromen Mosaik ausgestattet, dessen Oberkante 0.1-0.12 m über der Höhe des östlichen Stylobaten liegt. Die Treppe im Osten nach MR07 existierte bei der Herstellung des Mosaiks bereits, dieses wurde im Norden an die Apsis angepaßt und im Westen gegen eine Säulenbasis verlegt. Längliche, auf den Oststylobat versetzte Marmorblöcke, die südlich an die Basis anschließen, fassen seinen Rand ein. Das Mosaik wurde von Werner Jobst vollständig freigelegt und publiziert ${ }^{31}$. Es sind zwei Felder zu erkennen, die beide von einem weißen Randfeld eingefaßt werden. Das im Norden liegende, fast vollständig erhaltene Figurenfeld, das eine Gelageszene wiedergibt, nimmt Bezug auf die Apsis; das südlich anschließende, geometrische Feld des Mosaiks ist im Süden abgebrochen. Das Mosaik war etwa $3.2 \mathrm{~m}$ breit, es reicht im Süden bis MR16 III, ist also noch etwa $4.8 \mathrm{~m}$ lang.

\footnotetext{
30 s. Abb. 6, Bauteile, die sich aus dieser Phase erhalten haben, sind grün wiedergegeben.

31 Jobst (Anm. 5) 73 ff.; s. V. Scheibelreiter, Studien zu den kaiserzeitlichen Mosaiken Westkleinasiens (ungedr. Diss. Wien 2001).
} 
Bereits Franz Miltner, der den Befund als »Badebecken für das gemeinsame Bad der Liebespaare ${ }^{32}$ deutete, ging davon aus, daß der Raum auch im Süden »rund abgeschlossen « war. Werner Jobst rekonstruierte die ursprüngliche Länge des Raumes, dessen südliches Ende in einer nachfolgenden Phase vom Stiegenaufgang in MR16 überbaut wurde, mit $8.5 \mathrm{~m}^{33}$. Dieser Rekonstruktion ist prinzipiell zuzustimmen, da die Komposition des Mosaiks nach einem Pendant zu dem nördlichen Figurenfeld verlangt, das vor den Stufen gelegene, geometrische Feld mag als Achse fungieren. Von einer zweiten Apsis, die im Süden das Mosaik abgeschlossen hat, ist daher auszugehen. Diese Annahme wird durch den Befund eines Hinterfüllmörtelrestes an der Ostwand von MR16 bestätigt, der zu einer Sockelverkleidung gehörte, wie sie an der Nordapsis und dem anschließenden Mauerrest an der Ostwand des Raumes MR10a bis zur Treppe nachzuweisen ist.

Was die Nutzung des Saales betrifft, so sprach sich Jobst ${ }^{34}$ für einen Speisesaal aus. Sieht man den Raum jedoch im Kontext des Peristyls und der östlich anschließenden, großzügigen Raumgruppe, so kommt dem apsidialen Raum vielmehr eine Funktion als Bindeglied zu, das den straßenseitigen, westlichen Bereich elegant mit dem abgelegenen, östlichen verbindet. Bedenkt man, daß die Öffnung in der Ostwand des Apsidensaales in dieser Phase die einzige Zugangsmöglichkeit zu den östlichen Räumen darstellte, so kann der Saal auch als Zwischenraum, der durch seine Ausstattung auf die Funktion der östlichen Räume hinweist, gedeutet werden (Abb. 6).

In der dritten Phase wurden die Räume, abgesehen vom Apsidensaal, nicht mehr mit Plattenverkleidungen ausgestattet, die neuerrichteten Süd- und Westwände ${ }^{35}$ von MR07 scheinen gar ohne Ausstattung geblieben zu sein. Die Wände von MR04 hingegen wurden verputzt, an der Nordwand von MR04 ist links und rechts der Türöffnung zu MR07 die Imitation einer Wandverkleidung zu erkennen. Die in der dritten Phase entstandene Mauer war an ihrer Südseite also verputzt und bemalt, während ihre Nordseite roh belassen wurde. Die Wandverkleidung des Raumes MR08 wird von einer Malerei, die eine dreizonige Marmorausstattung imitiert, abgelöst (Abb. 10. 11).

Der Grundriß des 'Peristyl-Atrium-Hauses' wird in der dritten Phase nicht aufgelöst. Die Bedeutung der östlichen, anläßlich der Hauserweiterung der zweiten Phase gewonnenen Raumgruppe wird durch den Einbau des im Ostgang des Peristyls gelegenen Apsidensaales verstärkt, der Saal selbst wird zum zentralen Gestaltungselement, das die westliche und östliche Haushälfte miteinander verbindet.

\section{Phase: Haus 'A' und 'B', Zerfall}

Die an diese drei Phasen anschließende Umgestaltung des Hauses führt zu seiner Auflösung. Im Rahmen der Bauuntersuchung wurden zumindest zwei weitere Phasen festgestellt, die an das Ende der baulichen Entwicklung des Mittelteiles der Insula M/1 zu setzen sind (Abb. 7) ${ }^{36}$; sie werden hier zusammengefaßt dargestellt. Das Ende der Nutzung der Bausubstanz ist von zwei einschneidenden Zerstörungen geprägt, die östlichen Bereiche des 'Peristyl-Atrium-Hauses' wurden dabei teilweise verschüttet und nicht mehr auf ursprünglichem Bodenniveau genützt. Unzugänglich blieben die Räume MR07 und MR07a sowie MR08 und MR08a, ihre Nutzung

\footnotetext{
32 Miltner (Anm. 3) 320.

33 Jobst (Anm. 5) 84. Die Rekonstruktionszeichnungen geben die Dimensionen der Raumgruppe m. E. allerdings nicht korrekt wieder.

34 Jobst (Anm. 5) 82.

35 Angesprochen wird hier die Ostseite der Mauer, an ihrer Westseite hingegen wurde eine Plattenverkleidung, zur Sockelzone des Apsidensaales gehörig, angebracht!

36 s. Abb. 7, erhaltene Bauteile aus diesen beiden letzten Phasen sind gelb angegeben. Die Nordseite des rekonstruierten 'Peristyl-Atrium-Hauses' wird einem anderen Haus zugeschlagen und ist daher hier nicht mehr dargestellt. Die neue nördliche Hausbegrenzung verläuft entlang der ehemaligen nördlichen Hofwand des Peristyls.
} 
endet mit der dritten Phase. Das Peristyl und der Apsidensaal verschwinden. Das Haus wird entlang des alten Südstylobaten des Peristyls geteilt, die Bereiche südlich werden einer geschlossenen Verbauung, die bis an die Kuretenstraße heranreicht, zugeschlagen ('Haus A') ${ }^{37}$. Die Räume nördlich dieser neu eingezogenen Hausgrenze können nur mehr von Norden her, abgesehen von den weiterhin bestehenden Tabernen, nicht mehr aber von der Marmorstraße aus betreten werden. Sie sind ihrerseits Teil einer neuen, in sich geschlossen genutzten Raumgruppe ('Haus B') ${ }^{38}$.

\section{'Haus $A$ '}

Der verschüttete Raum MR07 wurde weiterhin genutzt, allerdings auf deutlich höherem Niveau, das eventuell dem des Obergeschosses angeglichen war, der Zugang erfolgte über eine Treppe, die in MR16 liegt. Die Räume MR04 und MR05 sowie MR06 wurden nutzbar gehalten; hiezu brach man eine Türöffnung in die Westmauer von MR04. Der unbrauchbar gewordene Eingang zu MR07 wurde mit einer schmalen Ziegelmauerung zugesetzt, der einstige Zugang, der von MR07 nach MR05 führte, durch den Einbau von Wandnischen vermauert. Über MR05 entstand ein zweistufiges Gewölbe. Die Wände von MR04 und MR05 wurden mit neuem Wandverputz versehen, auf den eine einfach gehaltene, rote Streifenmalerei aufgetragen war. Beide Räume wurden, wie oben bemerkt, über eine in die Westwand von MR04 gebrochene Türöffnung zugänglich gemacht, welche fest verschlossen werden konnte. Zeugnis geben Einlassungen für einen Sperrbalken in den Türgewänden. Die Art der Anlage und Umgestaltung deutet auf eine werkstättenähnliche Nutzung des Bereiches hin. MR06 scheint über eine Treppe vom Obergeschoß her wieder nutzbar gemacht worden zu sein, somit stand auch einer fortgesetzten Verwendung des Kellers unterhalb des Raumes nichts im Wege. Der Raum MR03 blieb von diesen baulichen Veränderungen unberührt. An der Schnittstelle der beiden Nutzungseinheiten, der 'Werkstätte' und des erhöhten Raumes MR07, wurde ein Hof angelegt, der als Verteiler fungierte. Er durchbrach die ursprüngliche südliche Hausgrenze, die in weiterer Folge allerdings wieder aufgenommen wurde. Der Zugang zu den voneinander abgesetzten beiden Einheiten konnte von Süden wie auch von Westen her erfolgen.

\section{'Haus $B$ '}

Die nördlich der neu eingezogenen Hausgrenze gelegenen Räume werden, wie oben erwähnt, einem separaten Haus zugeschlagen, die Stichgasse im Norden wird im Zuge dieser neuen Raumaufteilung überbaut. Der Raum MR09, an der Nordostecke des alten Peristylhauses (Phase 1) gelegen, bleibt weiterhin auf etwa gleichem Bodenniveau nutzbar, selbiges gilt auch für den Bereich des einstigen Nordganges des Peristyls (MR11). Diese nördliche Einheit scheint, wie jene von 'Haus A', im Kontext handwerklicher oder gewerblicher Nutzung gestanden zu sein; darauf lassen in den Boden eingelassene Dolia schließen ${ }^{39}$. Die Räume von 'Haus B' konnten einerseits durch die Taberne (MR14), andererseits über zwei Zugänge an der Nordseite betreten werden.

Die Mauern der letzten Phasen werden weiterhin überwiegend in opus mixtum-Technik hergestellt. In den Steinreihen finden sich recht sorgfältig zugerichtete Spolien, die Ziegelausgleichslagen sind ein- bis zwei-, seltener dreilagig. Dem Fugenmörtel wird meist Serecit- und/ oder Ziegelsplitt zugeschlagen, die Fugen werden verstrichen. Erstmals werden Mauern auch gegen Schutt errichtet ${ }^{40}$. Ein zweiter, gleichzeitig vorkommender Mauertyp besteht aus ungleich starkem Mauerwerk, dessen Oberfläche einer Glättung mit großzügigem Mörteleinsatz ${ }^{41}$ bedurfte. Es besteht im weitesten Sinn aus opus mixtum, die Ausgleichslagen orientieren sich aber nach

\footnotetext{
37 s. Abb. 7, weiß belassene Bereiche.

38 s. Abb. 7, grau gekennzeichnete Bereiche.

39 s. T. Bezeczky, The Laecanius Amphora Stamps and the Villas of Brijuni, DenkschrWien 261 (1998) $33 \mathrm{ff}$. Abb. 27.

40 s. Abb. 1. 2, Rückseiten der Nischen in der Nordwand von MR05 sowie Westwand von MR10.

${ }^{41}$ s. Abb. 1. 2, Südwand von MR10.
} 
den Größen der (nicht oder wenig zugerichteten) verbauten Spolien. Der Fugenmörtel ist kalkarm, die Zuschlagstoffe sind grobkörnig. Im Zuge von weiteren baulichen Veränderungen wurden verstärkt Ziegel, besonders in sekundärer Verwendung, zur Herstellung von Mauerwerk verwendet, dem Fugenmörtel wurde dann immer grober Ziegelsplitt beigemengt, die Wände blieben oftmals unverputzt.

In der anschließenden Entwicklung des Mittelteiles der Insula M/1 zeigt sich eine fortschreitende Zergliederung der Bausubstanz, die zuvor eingezogene Hausgrenze wurde mit einer Stiegenanlage ${ }^{42}$ überbrückt, die Zugänge, die von Norden her zu 'Haus B' führten, wurden zugemauert. Die Bodenniveaus von 'Haus B' wurden angehoben, während jene von 'Haus A' unverändert blieben.

\section{Schlußbetrachtung}

Zusammenfassend ergibt sich folgendes Bild: In der ersten Phase der Verbauung des Mittelteiles der Insula M/1 bestand ein nahezu quadratisches Peristylhaus von etwa $742.5 \mathrm{~m}^{2}$ Grundfläche. Der Hauptzugang erfolgte über die Marmorstraße, das im Zentrum des Hauses gelegene Peristyl war an seinen vier Seiten von Umgängen, an die Räume anschlossen, umgeben. In der zweiten Phase wurde das Haus nach Osten hin erweitert, die neugeschaffenen Räume wurden tief einschneidend in den Hangfuß des Panayırdağ errichtet, die Grundfläche erhöhte sich dadurch auf $879.5 \mathrm{~m}^{2}$. Eine zentrale Raumgruppe wurde symmetrisch in der Achse des Peristyls angelegt, nördlich und südlich dieser Raumgruppe wurden begleitende Räumlichkeiten geschaffen. Die Räume waren aufwendig ausgestattet und lassen auf eine repräsentative Verwendung schließen. In der folgenden dritten Phase wird einem offenbar noch gestiegenen Repräsentationsbedürfnis Rechnung getragen. Durch den Einbau eines Apsidensaales wird die im Zuge der zweiten Phase hinzugewonnene Bausubstanz gegen die der ersten Phase abgegrenzt, die räumliche Wirkung der Anlage so verstärkt. Teilweise werden nun Wandverkleidungen durch Malereien ersetzt. In der vierten Phase kommt es nach Zerstörungen zur Auflösung des 'Peristyl-Atrium-Hauses'; entlang einer Ost-West-Achse, die etwa durch die Mitte des ursprünglichen Hauses verläuft, entstehen, teilweise auf Schutthorizonten errichtet, die Häuser 'A' und 'B'. Nun kann der Beginn einer handwerklichen Nutzung der Bereiche nachgewiesen werden. In weiterer Folge wurden die zuvor klar voneinander abgegrenzten Häuser ' $A$ ' und 'B' teilweise wieder miteinander verbunden und bestehende Räume weiter verkleinert.

Mag. Dimitrios Boulasikis

c/o Österreichisches Archäologisches Institut, Franz Klein-Gasse 1, A-1190 Wien

E-Mail:dimitrios.boulasikis@oeai.at

Abbildungsnachweis: alle Abb. Verf.

42 s. Abb. 1. 2, MRA. 